\title{
Correspondence
}

\section{Cerebral blood flow velocity variability in infants receiving assisted ventilation}

Sir,

We read the article by Rennie et al with great interest. ${ }^{\prime}$

In the absence of any recording of blood pressure it is not possible to say whether perturbations in cerebral blood flow velocity result from transmission within the arterial system, or from the effects of changes in cranial venous volume, which are also known to affect it. ${ }^{2}$ Unfortunately, as these different effects tend to be opposite in direction and out of phase with each other, it is not possible to distinguish between them by an analysis of cerebral blood flow velocity and respiratory phase alone. The lack of distinction between the two effects is underlined by a failure to find a difference in the coefficient of variation expressed as a percent $(\mathrm{CV} \%)$ of the spectrum area between the apnoeic and synchronously ventilated groups. This is the more surprising as it is known that the effects of passive lung inflation $v$ spontaneous inspiration on left ventricular output and intracranial venous volume are not only opposite in direction, but also quite different in magnitude. Our own work suggests that the $\mathrm{CV} \%$ is inaccurate at low levels of variability such as those encountered during muscle paralysis and ventilation. ${ }^{3}$

Another potential inaccuracy in the use of the $\mathrm{CV} \%$ of the spectrum area lies in its reliance on the relative frequencies of respiration and heart rate; if spontaneous respiratory effort is occurring irregularly or infrequently, then the duration of 10 cardiac cycles may not be sufficient to quantify the effects of this activity on cerebral blood flow velocity. We have found good correlation between the CV\% over 20 cardiac cycles (used in Perlman's original work) and an estimate of respiration induced variability using spectral analysis, at least in aortic blood pressure. during spontaneous respiration. ${ }^{3}$

Perhaps the most important effect of any treatment designed to stabilise the pattern of respiration in the first days of life is to prevent episodes of crying. Valsalva manoeuvres, and pneumothoraces which can affect both intracranial venous volume and arterial blood pressure adversely.

\section{References}

' Rennic JM. South M. Morley CJ. Cerebral blood flow velocity variability in infants receiving assisted ventilation. Arch Dis Child 1987:62:1247-51

2 Cowan F. Thoresen M. The effects of interruption to the cranial venous drainage on ecrebral haemodynamies in the newborn infant. In: Rolfe P. ed. Neonatal physiological measurements. London: Butterworth. 1986.

${ }^{3}$ Bignall S. Bailey PC. Rivers RPA, Lissauer TJ. Quantification of cardiovascular instability in premature infants using spectral analysis of waveforms. Pediatr Res (in press).

$\mathrm{S}$ Bignal.t. and RPA Rivers St Mary's Hospital Medical School, London W2 INY
Drs Rennie and Morley comment:

We thanks Drs Bignall and Rivers for their interest in our paper. Our present system allows the collection of only six seconds' worth of Doppler information, which was the reason for choosing 10 cardiac cycles, but the spectrum generated was from a fast Fourier transform and a Duplex system, which may bear more relationship to velocity than the continuous wave system used by Perlman and Volpe. While accepting that the area under the curve measured with computer assisted planimetry is a relatively crude index of variability the method was sensitive enough to detect differences between synchronous and asynchronous ventilation and the values for variation are in good agreement with other estimates of variability in systemic arterial pressure. ${ }^{2}$ We feel that the protocol, in which each infant acted as his or her own control and the velocity was measured with exactly the same technique in both types of ventilator interaction, adds confidence to what was a highly significant result $(\mathrm{p}<0 \cdot 001)$.

Further work is undoubtedly required on the underlying causes of variability and its accurate detection, and on the possible benefits of non-triggered synchronous ventilation. Changes in central venous pressure are important and have been shown to occur sometimes opposite to the systemic arterial pressure swings, meaning that the changes in cerebral perfusion pressure are more marked. ${ }^{1}$ We plan to study this interesting phenomenon further with an improved method allowing rapid computer estimation over several minutes.

\section{References}

1 Perlman JM. Volpe JJ. Are venous circulatory abnormalities important in the pathogenesis of cerebral Iesions? Pediatrics 1987:80:705-11.

- Miall-Allen V. De Vries L. Whitelaw A. Mean arterial blood pressure and neonatal cerebral lesions. Arch Dis Child 1987:62:1068-9.

$\mathrm{J}$ RINNIE and C MORLEY Department of Paediatrics, University of Cambridge Clinical School, Level 8. Addenbrooke's Hospital. Cambridge CB2 $2 Q Q$

\section{Management of sexual abuse}

Sir,

Drs Hobbs and Wynne stress the importance of reflex anal dilatation as a sign of anal sexual abuse. ${ }^{1}$ In their reply to Dr Robert's scepticism concerning the reliability of the observation, they state 'We hold by our view that reflex dilatation of the anus correlates highly with continuing abuse .... and is not found in diseases such as thrush. threadworms, or constipation'. While not wishing to dispute this statement entirely, we do not believe that 
reflex anal dilatation occurs exclusively in cases of sexual abuse.

In a recent experience at this hospital, a 7 year old boy was admitted with non-specific abdominal pain, vomiting, and perianal soreness. Physical examination showed reddening of the anus, an anal fissure, and marked reflex anal dilatation; a diagnosis of sexual abuse was considered on the basis of these findings. There were no other features to support the diagnosis, however, and the child seemed a cheerful, well adjusted boy who enjoyed an excellent relationship with his parents.

Before voicing our suspicions, further questioning showed recent onset of mild diarrhoea containing mucus. A barium follow through examination subsequently showed extensive changes of Crohn's disease in the terminal ileum. Colonoscopy confirmed mucusal ulceration in this region, and histology of a biopsy specimen was consistent with Crohn's disease. The child was started on an elemental diet, and his symptoms and signs resolved quickly.

Drs Hobbs and Wynne point out that they never base their diagnoses of abuse on the anal dilatation reflex alone, but in conjunction with other signs of anal damage such as . . . fissures, veins, thickened perianal skin, scars . . . etc.' These abnormalities, however, are all common findings in the $46 \%$ of children with Crohn's disease who have anal involvement at time of presentation. ${ }^{2}$ Presumably, reflex anal dilatation occurs in some of these children in response to the pain often associated with perianal lesions in Crohn's disease.

This case emphasises the importance of not relying on anal signs alone when diagnosing child sexual abuse. Crohn's disease occurs in approximately 1 child per 10000 , and, although uncommon, does appear to be increasing in incidence. ${ }^{2}$ It would, therefore, benefit paediatricians and general practitioners to bear the condition in mind when diagnosing child sexual abuse on the basis of abnormal anal findings.

\section{References \\ 1 Hobbs CJ, Wynne JM. Management of sexual abuse. Arch Dis Child 1987:62:1182-7; comment:1195. \\ 2 Sanderson IR. Chronic inflammatory bowel discase. Clin Gastroenterol 1986;15:71-87. \\ C M Evans and $\mathrm{J}$ A Walker-Smith Department of Paediatric Gastroenterology, St Bartholomew's Hospital, London ECIA $7 B E$ \\ Anal dilatation and anal dilatation reflex associated with severe haemorrhagic colitis}

\section{Sir,}

There is no doubt that dilatation of the anus and the anal dilatation reflex can be associated with penetrating anal abuse. Recent controversy has focused on these two features, particularly the latter, among the many manifestations of child sexual abuse. Clearly doubt exists regarding the validity of these two signs as sole indicators of anal sexual abuse in children. They have been described also in Crohn's disease ${ }^{1}$ and constipation (the passage of a very large formed stool). ${ }^{2}$ It remains part of the controversy whether other local irritant or inflammatory conditions such as threadworm and rectal candidiasis can be associated with these signs. ${ }^{2}$;

We recently admitted a toddler, aged 13 months, with a three day history of watery diarrhoea. For the two days before admission his stools were bloody and frequent, he was lethargic and unwell but not febrile. He had not received any medication, and his parents denied administering rectal preparations or using rectal thermometers. His bowel motions had previously been regular and soft, without pain or bleeding.

On examination the perianal skin was reddened, and the anus was $2 \mathrm{~cm}$ patent, showing red anal and rectal mucosa with oedema blurring the pectinate line. Gently parting the buttocks resulted in reflex anal dilatation by approximately a further centimeter. There were no abnormal neurological signs. Careful questioning of both parents independently yielded the same story: the anus had become patulous on the previous day, from which time his diarrhoea had been 'running out of him'.

During his admission he developed the colitis associated haemolytic uracmic syndrome. No pathogens or toxins were isolated from his stools, although it is thought that most patients with this form of the disease have been infected with verotoxin-producing Escherichia coli. Six weeks later his anus was entirely normal, and remained so on a subsequent admission.

We conclude that rectal inflammation may be associated with unexpected dilatation of the anus and the anal dilatation reflex. It would be pertinent to ask how little (or how much) inflammation or irritation is required?' and, 'for how long after the inflammation has clinically settled can these signs persist?'

\section{References \\ I Hey F. Buchanan PC. Litllewood JM. Hall RI. Differential diagnosis in child sexual abusc. Lancet 1987:i:283. \\ 2 Clayden G. Anal appearances and child sex abuse. Lancel 1987:i:620-1. \\ "Hobbs CJ. Wynne LM. Management of sexual abuse (Com- ment). Arch Dis Child 1987:62:1195.}

A R Magnay and J Insley Birmingham Children's Hospital, Ladywood Middleway. Birmingham $B I 0 \quad 8 E T$

\section{Immunisation in the immunosuppressed child}

Sir,

We read with interest the annotation on immunisation in immunosuppressed patients. 'The author highlighted the problems associated with viral infections and the need to determine immune state. Chickenpox is a common infectious illness which can lead to major complications in immunosuppressed children. Unfortunately previous illness 